\title{
Analysis of Stored Energy in Cold-Rolled Copper Using Bulk and Microstructure-Based Techniques
}

\author{
Shan-Quan Deng ${ }^{1} \cdot$ Andy Godfrey $^{1} \cdot$ Wei Liu ${ }^{1}$
}

Received: 3 November 2015/Revised: 14 December 2015/Published online: 26 February 2016

(C) The Chinese Society for Metals and Springer-Verlag Berlin Heidelberg 2016

\begin{abstract}
Measurements of stored energy have been obtained for samples of copper cold-rolled to von Mises strains between 0.42 and 5.21 using both differential scanning calorimetry (DSC), and based on measurements of microstructural parameters in the transmission electron microscope (TEM). In both cases, a linear increase in stored energy with strain is found. The ratio between the two measured values varies, however, over a significant range, indicating that some caution is needed in determining the relative difference in energy associated with deformation microstructure heterogeneities in a given sample. Comparison of the stored energy with the flow stress suggests that the TEM-based measurements reflect the dislocation density content responsible for the flow stress, but that the DSC technique additionally measures other contributions to the stored energy, such as the presence of balanced internal stresses.
\end{abstract}

KEY WORDS: Stored energy; Differential scanning calorimetry; Microstructure; Flow stress; Copper

\section{Introduction}

During plastic deformation of metals, a small fraction of the total dislocation density activated during slip remains in the material, representing the stored energy of cold work $\left(E_{\mathrm{S}}\right)$. This corresponds typically to a few percent of the external work done in deforming the material $[1,2]$. In the case of metals with a medium to high stacking fault energy, these dislocations are stored predominantly in dislocation rotation boundaries, which increase in misorientation angle and decrease in spacing with increasing applied strain [3, 4], in both tension [5] and rolling [6], resulting in a parallel process of microstructural subdivision and increase in flow stress (i.e., work hardening). The relationship between the

Available online at http://link.springer.com/journal/40195.

Andy Godfrey

awgodfrey@mail.tsinghua.edu.cn

1 Key Laboratory of Advanced Materials (MOE), School of Materials Science and Engineering, Tsinghua University, Beijing 100084, China stored energy and flow stress has been investigated in study of aluminum deformed by cold rolling over a range of von Mises strain from $\varepsilon_{\mathrm{vM}}=0.06$ to $\varepsilon_{\mathrm{vM}}=2.65$ [7], where it was found that the flow stress was linearly related to $E_{\mathrm{S}}^{0.5}$ but with a different constant of proportionality for low strains $\left(\varepsilon_{\mathrm{vM}}<0.5\right)$ and for high strains $\left(\varepsilon_{\mathrm{vM}}>0.5\right)$.

The stored energy of cold work is also an important parameter for understanding, and control of, the thermal stability of deformed metals. For example, it is known that in deformed $\mathrm{Cu}$ of $99.9 \%$ purity, recrystallization may take place at room temperature [8], whereas this does not happen in $\mathrm{Al}$ of similar purity, on account of the fact that more dynamic recovery takes place during the deformation of $\mathrm{Al}$, so that only a smaller part of the work of plastic deformation remains stored within the deformation microstructure.

More importantly, both nucleation of recrystallization and the migration of recrystallizing boundaries have been found to be strongly sensitive to local variations in stored energy of deformation. A clear example of this is the nucleation of recrystallized grains in shear bands, and the 
growth of these grains along the shear bands in both polycrystals [9] and single crystals [10]. Consequently, it is of interest to have good measures of both the global stored energy and of local variations in stored energy.

The former is most commonly measured using differential scanning calorimetry (DSC) [11], although other methods have also been used based on X-ray [12] or neutron [13] diffraction measurements. Local variations in stored energy, in contrast, rely on microstructural measurements, where the stored energy is calculated from the observed boundary spacing and misorientation angles of the dislocation boundaries within selected regions of the deformation microstructure. For such calculations, it is usually assumed that the energy per unit area of a dislocation boundary $(\gamma)$ depends on the boundary misorientation according to the Read-Shockley function. The energy per unit volume is then derived using a stereological relationship between the boundary spacing $(d)$ and the area per unit volume $\left(S_{\mathrm{V}}\right)$ of the boundaries of the form $S_{\mathrm{V}}=A / d$ [14], where $A$ is a constant that depends on the specific geometry of the boundaries. This calculation can be made using either average values, or using individual boundary measurements in which case the area per unit volume is derived from a direct measurement of the boundary length per unit area in the observation plane. The latter is more typical for calculations based on electron back scatter diffraction (EBSD) data (where other parameters such as the kernel average misorientation have also been used to estimate stored energy [15]). Microstructural data obtained in the transmission electron microscope (TEM) are, however, considered more reliable, as a result of the limited angular resolution of the EBSD technique, where boundary misorientations of less than $1.5^{\circ}-2.0^{\circ}$ in deformed samples cannot be reliably resolved [16]. Efforts to predict the stored energy of cold work have also been made on the basis of discrete dislocation plasticity calculations [17], where the effects of plastic dissipation and elastic energy can be separated. These methods are, however, most applicable to small strains (0.05-0.1 in [17]), where long range stresses are still considered to be significant, and where experimental measurements of stored energy are more challenging.

A key question, however, for microstructural-based estimates is the accuracy of the data. In a previous study, the difference in stored energy between local (TEM) and bulk (DSC) measurements has been examined for $\mathrm{Ni}$ deformed to strains above $\varepsilon_{\mathrm{vM}}=1$, where it was found that the two values (bulk and local estimates) are approximately proportionally related, but with the DSC value larger by a factor of $2-3[18,19]$. This difference was presumed to arise from the assumption that the stored energy is stored only in dislocation boundaries (ignoring, for example, the contribution of triple junctions) and that the dislocation boundaries are fully stress-screened (as assumed by the use of the Read-Shockley function for the relationship between boundary energy and misorientation angle). The approximately linear relationship between the DSC and microstructure-based values nevertheless gives some confidence to the analysis of relative differences in the stored energy for a given microstructure.

As the tendency for the well-defined formation of dislocation boundaries decreases with decreasing stacking fault energy, it is of interest to consider how both the relationship between DSC and microstructural estimates of stored energy, and the relationship between stored energy and flow stress are influenced by stacking fault energy. Here we investigate such relationships in cold-rolled copper, chosen as a metal with a stacking fault energy lower than both $\mathrm{Al}$ and $\mathrm{Ni}$, but where well-defined dislocation rotation boundaries are still known to form during plastic deformation [6].

\section{Experimental}

Commercial purity (99.9\%) oxygen-free high conductivity $(\mathrm{OFHC}) \mathrm{Cu}$, in a fully recrystallized condition with a grain size of $73 \mu \mathrm{m}$ (ignoring annealing twins) and a weak texture, was used for the investigations. The $\mathrm{Cu}$ sample, of initial size $41 \mathrm{~mm}$ (height) $\times 46 \mathrm{~mm}$ (width) $\times 100 \mathrm{~mm}$ (length), was cold-rolled to reductions from 31 to $99 \%$, corresponding to von Mises strains $\left(\varepsilon_{\mathrm{VM}}\right)$ from 0.42 to 5.21. The ratio of contact length to sample thickness during cold rolling was kept within the range from 0.5 to 5 , in order to ensure homogeneous deformation during rolling. As such, the reduction per pass was not uniform, but varied from approximately $4 \%$ to approximately $36 \%$. The total number of passes used to achieve each strain level is indicated in Table 1. A fixed roller speed was used, resulting in a variation in strain rate during rolling from 0.3 to $2.3 \mathrm{~s}^{-1}$. At high accumulated strains, the sample was rapidly cooled in water immediately after each rolling step, in order to prevent recrystallization and to inhibit as far as possible any post-deformation recovery of the microstructure. All the cold-rolled samples were stored in a freezer at $-18{ }^{\circ} \mathrm{C}$ prior to the experimental investigations.

The deformation microstructure of $\mathrm{Cu}$ after rolling was investigated in the TEM in thin foil samples prepared from the longitudinal section (the plane containing the rolling direction and the normal to the rolling plane). The key microstructural parameters, namely the spacing and misorientation angle of dislocation boundaries, were measured in the TEM after tilting the foil so that the electron beam was nearly parallel to a low-index zone axis (typically $<110>$ ), thereby ensuring that all dislocation boundaries in the area to be examined were in contrast and clearly visible. 
Table 1 Microstructural parameters of IDBs and GNBs measured in TEM

\begin{tabular}{llllllll}
\hline$\varepsilon_{\mathrm{vM}}$ & Number of passes & $D^{\mathrm{IDB}}(\mu \mathrm{m})$ & $D^{\mathrm{GNB}}(\mu \mathrm{m})$ & $\theta^{\mathrm{IDB}}\left({ }^{\circ}\right)$ & $\theta^{\mathrm{GNB}}\left(\theta<15^{\circ}\right)\left(^{\circ}\right)$ & $f^{\mathrm{GNB}}\left(\theta<15^{\circ}\right)$ & $N^{2}$ \\
\hline 0.42 & 4 & 0.71 & 0.77 & 1.3 & 3.2 & 1 & 1 \\
0.78 & 6 & 0.60 & 0.45 & 1.6 & 3.5 & 6.0 \\
1.36 & 8 & 0.50 & 0.42 & 2.6 & 5.2 & 0.99 & 0.83 \\
2.72 & 12 & 0.43 & 0.20 & 3.2 & 5.6 & 0.74 \\
3.43 & 16 & 0.34 & 0.18 & 2.8 & 5.2 & 11.7 \\
5.21 & 37 & 0.29 & 0.15 & 2.9 & 6.7 & 11.2 \\
\hline
\end{tabular}

Bulk measurements of the stored energy in $\mathrm{Cu}$ after rolling were obtained using a NETZSCH STA 449 F3 differential scanning calorimetry (DSC) instrument. Before DSC measurement, each $\mathrm{Cu}$ sample (mass between 24 and $230 \mathrm{mg}$ ) was ground to 4000 grit $\mathrm{SiC}$ on all surfaces. For the DSC measurements, the $\mathrm{Cu}$ samples were heated from 323 to $873 \mathrm{~K}$ at a heating rate of $20 \mathrm{~K} \mathrm{~min}^{-1}$ in an argon atmosphere. Each sample was heated twice consecutively such that two DSC curves were recorded for each sample. The DSC curve representing the heat release due to the stored energy of deformation was obtained by subtracting the second curve from the first curve. The stored energy of each sample was calculated as the area of the heat release peak in this obtained DSC curve.

The flow stresses, $\sigma_{0.2 \%}$, of the cold-rolled $\mathrm{Cu}$ samples were obtained by uniaxial tensile testing. The tensile samples were of dog-bone shape, with a gauge length of $8 \mathrm{~mm}$, a width of $2 \mathrm{~mm}$ and a thickness of $1 \mathrm{~mm}$, and tensile testing was carried out using a displacement rate of $0.24 \mathrm{~mm} \mathrm{~min}^{-1}$, corresponding to an initial strain rate of $5.0 \times 10^{-4} \mathrm{~s}^{-1}$.

\section{Results}

A key feature found from the TEM observations is that over the entire strain range examined the deformation microstructure can be described by a cell-block structure, consisting of geometrically necessary boundaries (GNBs) and incidental dislocation boundaries (IDBs) [3-6], evolving from a microband-dominated structure at low to medium strains [6], to a fully lamellar microstructure at high strains [20]. Figure 1 shows example TEM images at low $\left(\varepsilon_{\mathrm{vM}}=0.42\right)$, medium $\left(\varepsilon_{\mathrm{vM}}=1.36\right)$, and high $\left(\varepsilon_{\mathrm{vM}}=3.43\right)$ strains. In each case, long straight boundaries can be classified as GNBs with the short interconnecting boundaries between these classified as IDBs. It is apparent that over the entire strain range examined some volumes between GNBs and IDBs contain some loose dislocations, while some volumes do not. In all cases though the density of these loose dislocations is considered to be low enough to ignore in comparison with those stored in dislocation boundaries, in line with similar studies of both $\mathrm{Al}$ [21] and Ni [20].

The microstructural parameters of these GNBs and IDBs, namely the spacing between the boundaries and the misorientation angle across the boundaries, were both measured from the TEM investigations in samples prepared from the longitudinal section. The average spacing of the GNBs $\left(D^{\mathrm{GNB}}\right)$ at each strain was calculated from measurements of the distance between neighboring GNBs measured along the direction perpendicular to the GNBs. Similarly, the average spacing of the IDBs $\left(D^{\mathrm{IDB}}\right)$ was determined from measurements of the spacing between neighboring IDBs, along the direction parallel to the surrounding GNBs. As shown in Fig. 1d, the ratio $N$, defining the aspect ratio of each measured cell block, was also measured. The use of this measurement is described further below. Average measurements of $D^{\mathrm{GNB}}, D^{\mathrm{IDB}}$ and $N$ at each strain were obtained from TEM measurements taken in more than seven (typically more than ten) regions from at least two separate TEM foils, to ensure a good statistical representation of the average microstructural values at each strain. The orientations of the volumes enclosed by individual IDBs and GNBs were determined in the TEM by the use of a semiautomatic method based on Kikuchi pattern analysis, from which the average misorientation angles across the GNBs and IDBs were calculated [22].

From the microstructural parameters determined from the TEM investigations, an estimation of the stored energy based on local microstructure can be made. The basis of this estimation is an assumption that the stored energy is given by the energy of the dislocation boundaries stored in the material after deformation [7] where the geometry of the dislocation boundaries is considered [14]. Accordingly for an accurate calculation, it is necessary to consider the contribution to the stored energy of the GNBs and IDBs separately. As both the IDBs and GNBs are regarded as low-energy dislocation boundaries, the energy per unit area of the boundaries $(\gamma)$ can be estimated for each according to the Read-Shockley equation [23]: 

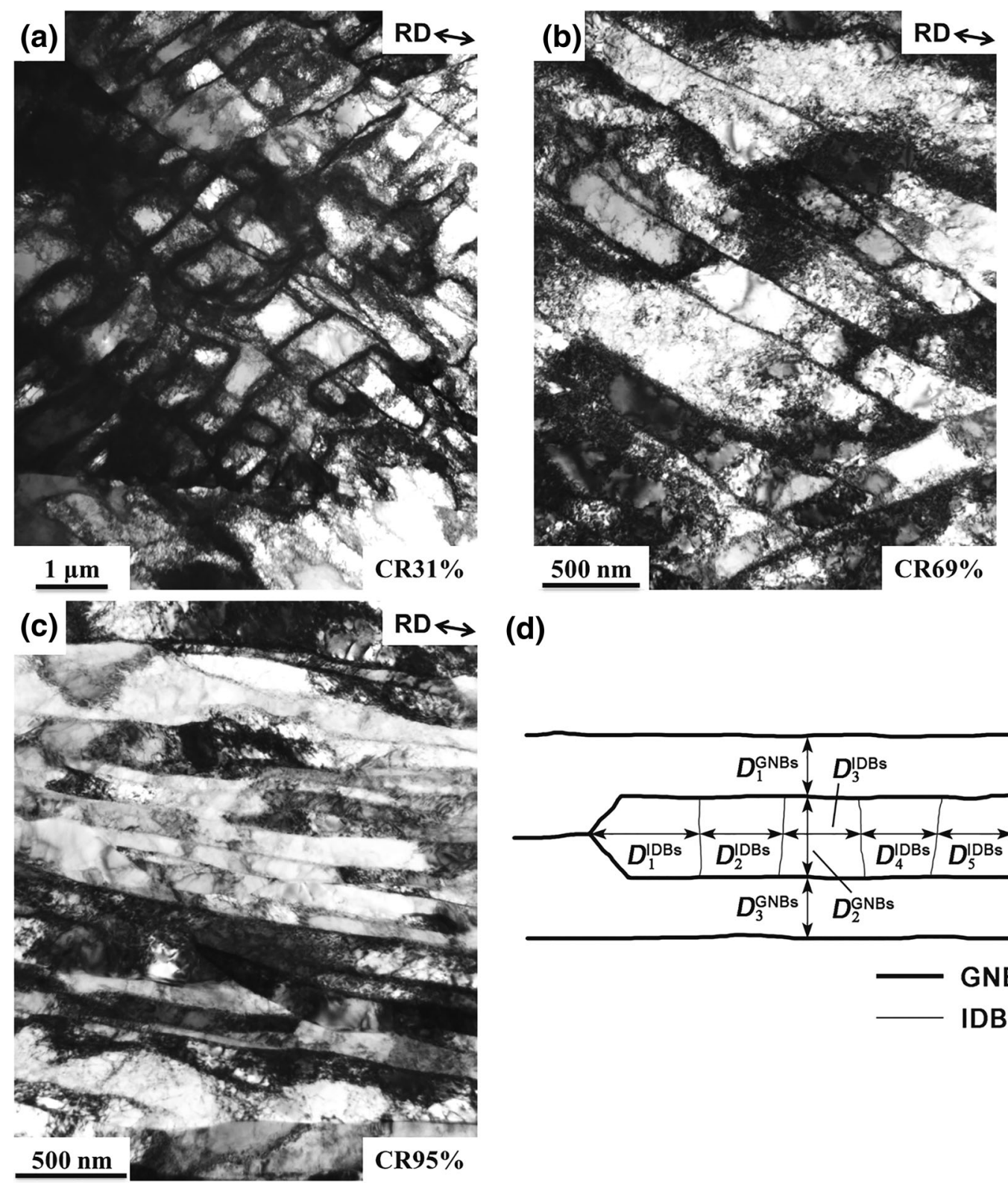

(d)

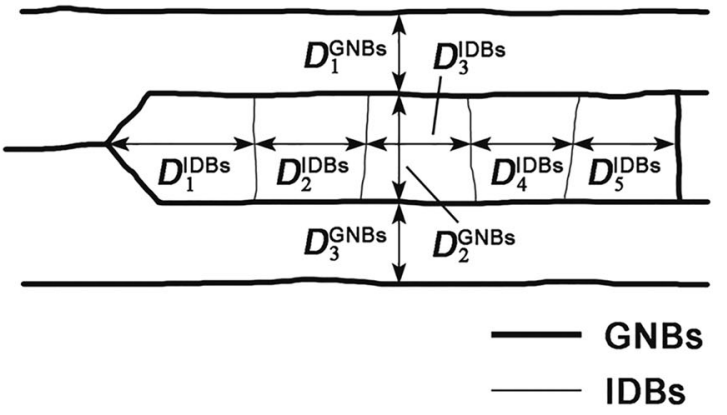

Fig. 1 Example TEM images showing typical dislocation cell-block morphologies in samples of Cu cold-rolled to strains of $0.42 \mathbf{a}, 1.36 \mathbf{b}$ and $3.43 \mathbf{~}$; $\mathbf{d}$ schematic showing the approach used for determination of the dislocation boundary data from the TEM observations. In this case, three individual GNB spacings $\left(D_{1-3}^{\mathrm{GNB}}\right)$ and five individual IDB spacings $\left(D_{1-5}^{\mathrm{IDB}}\right)$ are measured. For the cell block shown here the aspect ratio, $N$, is given by $\operatorname{SUM}\left\{D_{1-5}^{\mathrm{IDB}}\right\} / D_{2}^{\mathrm{GNB}}$

$\gamma=\gamma_{\mathrm{m}}\left(\theta / \theta_{\mathrm{m}}\right)\left[1-\ln \left(\theta / \theta_{\mathrm{m}}\right)\right]: \quad \theta<\theta_{\mathrm{m}}$,

$\gamma=\gamma_{\mathrm{m}}: \quad \theta \geq \theta_{\mathrm{m}}$,

where $\gamma_{\mathrm{m}}$ is the energy of a high angle boundary $\left(\theta \geq 15^{\circ}\right)$ per unit area $\left(0.625 \mathrm{~J} \mathrm{~m}^{-2}\right.$ for copper), $\theta$ is the boundary misorientation angle and $\theta_{\mathrm{m}}$ is the minimum angle to define a high angle boundary, taken here as $15^{\circ}$.

It can be noted that all the IDBs measured in this study had a misorientation angle smaller than $15^{\circ}$, whereas the range of GNBs misorientation angles extended to values larger than $15^{\circ}$. Therefore, the stored energy for the GNBs $\left(\gamma^{\mathrm{GNB}}\right)$ is divided into two parts according to Eqs. (1) and $(2)$. Accordingly, the stored energy $\left(E_{\mathrm{S}}\right)$ of copper during cold rolling can be calculated as:

$E_{\mathrm{S}}=S_{\mathrm{V}}^{\mathrm{IDB}} \gamma^{\mathrm{IDB}}+S_{\mathrm{V}}^{\mathrm{GNB}}\left[\gamma_{\theta<15^{\circ}}^{\mathrm{GNB}} f_{\theta<15^{\circ}}^{\mathrm{GNB}}+\left(1-f_{\theta<15^{\circ}}^{\mathrm{GNB}}\right) \gamma_{\mathrm{m}}\right]$,

where $S_{\mathrm{V}}^{\mathrm{IDB}}$ is the boundary area per unit volume of the IDBs, $\gamma^{\mathrm{IDB}}$ is the average energy per unit area of the IDBs, $S_{\mathrm{V}}^{\mathrm{GNB}}$ is the boundary area per unit volume of the GNBs, 
$\gamma_{\theta<15^{\circ}}^{\mathrm{GNB}}$ is the average energy per unit area of GNBs with $\theta<15^{\circ}$ and $f_{\theta<15^{\circ}}^{\mathrm{GNB}}$ is the fraction of GNBs with $\theta<15^{\circ}$. Based on the stereological characteristics of the cell-block structures formed during deformation, $S_{\mathrm{V}}^{\mathrm{IDB}}$ and $S_{\mathrm{V}}^{\mathrm{GNB}}$ can be calculated from the measurements of boundary spacing by the following equations.

$S_{\mathrm{V}}^{\mathrm{IDB}}=r_{\mathrm{IDB}} \pi /\left(2 D^{\mathrm{IDB}}\right)$,

$S_{\mathrm{V}}^{\mathrm{GNB}}=1 / D^{\mathrm{GNB}}+1 / D^{\mathrm{TRANSVERSE}}+1 / D^{\text {LONGITUDINAL }}$,

$r_{\mathrm{IDB}}=\left(D^{\mathrm{GNB}} N / D^{\mathrm{IDB}}-1\right) /\left(D^{\mathrm{GNB}} N / D^{\mathrm{IDB}}\right)$,

$D^{\text {TRANSVERSE }}=D^{\text {LONGITUDINAL }} \approx D^{\mathrm{GNB}} N$.

In these equations, $\gamma_{\text {IDB }}$ is a modified ratio that takes account of the fact that cell blocks are finite in length, and hence, the end of each cell block along the IDB measuring direction is formed in fact by a GNB rather than an IDB. This ratio is calculated using Eq. (6) based on the experimental measurements of $D^{\mathrm{GNB}}, D^{\mathrm{IDB}}$ and $N$, where $N$ is the measured aspect ratio of cell block. In Eq. (5), the values of $D^{\text {TRANSVERSE }}$ and $D^{\text {LONGITUDINAL }}$ are the calculated average spacing between GNBs along the transverse direction and longitudinal direction of each cell block. The factor of $\pi / 2$ in Eq. (4) takes account of the fact that IDBs have a random inclination to the measurement plane, whereas GNBs are observed typically in an edge-on condition (with the boundary plane perpendicular to the measurement plane). More details of the calculations concerning determination of the aspect ratio, $N$, and the relevance of the factor of $\pi / 2$ can be found in [20] and [24], respectively. The experimentally determined values used in the calculation of the stored energy are summarized in Table 1.

As average misorientation angles are used in calculating $\gamma^{\mathrm{IDB}}$ and $\gamma_{\theta<15^{\circ}}^{\mathrm{GNB}}$, Eq. (1) [23] in fact leads to an overestimation of the stored energy due to the nonlinearity of the Read-Shockley function. To correct this overestimation, a factor $\chi,\left(\chi_{\theta}^{\mathrm{IDB}}\right.$ for IDBs and $\chi_{\theta}^{\mathrm{GNB}}$ for GNBs), is used [7]. Accordingly, Eq. (3) can then be rewritten as

$$
\begin{aligned}
E_{\mathrm{S}}= & S_{\mathrm{V}}^{\mathrm{IDB}} \gamma^{\mathrm{IDB}} / \chi_{\theta}^{\mathrm{IDB}} \\
& +S_{\mathrm{V}}^{\mathrm{GNB}}\left[\gamma_{\theta<15^{\circ}}^{\mathrm{GNB}} \mathrm{G}_{\theta<15^{\circ}}^{\mathrm{GNB}} / \chi_{\theta}^{\mathrm{GNB}}+\left(1-f_{\theta<15^{\circ}}^{\mathrm{GNB}}\right) \gamma_{\mathrm{m}}\right] .
\end{aligned}
$$

The variation of stored energy $\left(E_{\mathrm{S}}\right)$ with strain, as estimated based on Eq. (8) using the experimental TEM measurements, is plotted in Fig. 2. An apparent linear relationship between the stored energy calculated based on microstructural parameters and strain is found, which is in good agreement with previous observations on cold-rolled Ni [18].

In addition to the estimate of stored energy based on the microstructural measurements, the stored energy on the

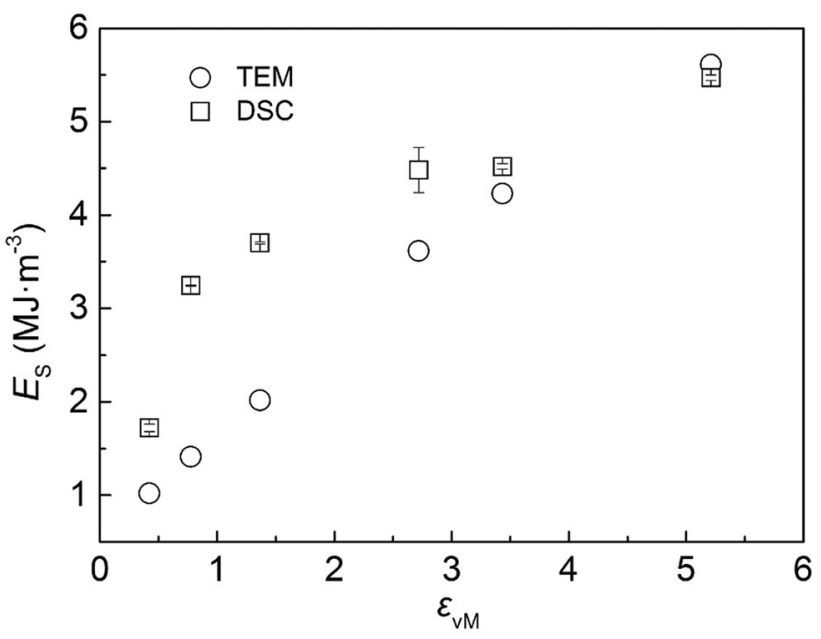

Fig. 2 Graph showing the variation of stored energy as a function of strain as determined from the TEM microstructural measurements and from the DSC data

bulk scale can be measured directly by the DSC technique [25]. The results of the DSC measurements of the stored energy for the cold-rolled $\mathrm{Cu}$ samples as a function of strain are also plotted in Fig. 2. Similar to the microstructural-based estimates of stored energy, it can also be found that the DSC-measured values of stored energy increase with increasing strain. Moreover, a linear relationship is also observed between the DSC-measured stored energy and strain, with the exception of the sample at the lowest strain.

\section{Discussion}

Both the DSC and the microstructure-based (local) measurements provide information about the stored energy of cold work on the bulk scale. For the DSC data, this comes directly from measurement on a bulk sample [25]. In the case of the microstructure-based estimate, an effective bulk value for the stored energy is obtained by ensuring that local measurements are made over a sufficiently large number of areas to represent accurately the average microstructural parameters. It is of interest therefore to examine how well the DSC and microstructural data agree with one another. Such a comparison is shown in Fig. 3, which plots the ratio of stored energy measured using the two techniques as a function of strain.

Two features of this plot are worth a special comment. The first is that the ratio $E_{\mathrm{S}, \mathrm{DSC}} / E_{\mathrm{S}, \mathrm{TEM}}$ decreases with increasing strain for all strains above $\varepsilon_{\mathrm{vM}}=0.78$ and moreover varies by a significant amount in the strain range examined. This is in contrast to observations of cold-rolled $\mathrm{Ni}[18,19]$. Although in the present study the microstructural measurements have been used to obtain an 


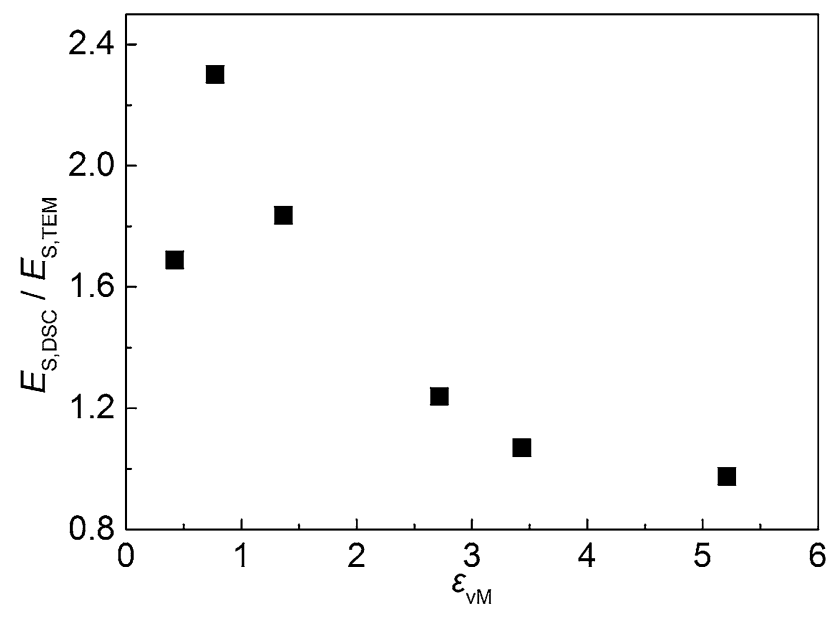

Fig. 3 Graph showing the ratio between the stored energy determined from the DSC data and from the TEM measurements as a function of strain

average bulk value of the stored energy, an important advantage of the microstructure-based method is that local variations in stored energy, corresponding to regions of heterogeneity in the deformation microstructure, which can differ significantly from global (bulk) values [17] can also be measured. On the assumption that the DSC data can be regarded as being more reliable (as the DSC measurements represent the average value over a larger volume of material), then the observed variation in the ratio of $E_{\mathrm{S}, \mathrm{DSC}} /$ $E_{\mathrm{S}, \mathrm{TEM}}$ implies that the relative variation in measured stored energy obtained from local microstructure-based estimates may not fully reflect the actual local relative variations. For $\mathrm{Cu}$, therefore, it is suggested that care should be taken in determining the relative value of stored energy made using local estimates, particularly when comparing regions of significant heterogeneity in the deformation microstructure.

A second point of interest in Fig. 3 is that the ratio of $E_{\mathrm{S}, \mathrm{DSC}} / E_{\mathrm{S}, \mathrm{TEM}}$ for cold-rolled $\mathrm{Cu}$ in this study is in general smaller than found for cold-rolled $\mathrm{Ni}[18,19]$, particularly at the highest strains, where the TEM data give a value very close to the DSC-determined stored energy. This gives good confidence to the absolute values of the local stored energy estimates obtained at each strain and suggest that the TEM measurements in this study provide a better absolute estimation of stored energy than in the previous study on Ni. The exact details of the sampling strategy used in the Ni study are not given, but one possible reason for the difference may be that in the current study special effort was made to sample a large number of IDBs, on the basis that in $\mathrm{Cu}$, particularly at lower strains, these are less well defined, and that a significant fraction of stored energy is associated, even at high strains, with boundaries of low misorientation angle [19].
Further insight into the reliability of the stored energy measurements can be obtained by considering the relationship between the stored energy and flow stress (also a sample scale measured property), based on tensile data for the samples determined post-rolling. The stored energy can also be related to the instantaneous flow stress during deformation, for example, using the methodology outlined in [17]. It can be noted here that the tensile tests used to determine the flow stress were carried out at a lower strain rate than used during rolling. Copper has, however, a low strain-rate sensitivity, and the flow stress measurements obtained from the tensile tests nevertheless reflect the structure on which the stored energy measurements were taken.

The stored energy can be regarded as proportional to the energy of a dislocation multiplied by the total dislocation density (assumed in this study to correspond to those stored in the GNBs and IDBs) [7].

$E_{\mathrm{S}}=K\left(G b^{2}\right) \rho$,

where $K$ is a constant representing the dislocation character, $G$ is the shear modulus, $b$ is the Burgers vector and $\rho$ is the dislocation density. Moreover, according to the Taylor model, the flow stress $\sigma_{0.2 \%}$ of a deformation microstructure is proportional to the square root of the dislocation density [7].

$\sigma_{0.2 \%}=M \alpha G b \rho^{0.5}$,

where $M$ is the Taylor factor and $\alpha$ is a constant with value about 0.24. Combining Eqs. (9) and (10) gives a relationship between flow stress and stored energy:

$\sigma_{0.2 \%} \propto E_{\mathrm{S}}^{0.5}$.

Based on this model, plots of $\sigma_{0.2 \%}$ versus $E_{\mathrm{S}}^{0.5}$ for both the stored energy estimated from TEM measurements and the stored energy from DSC measurements are shown in Fig. 4. For the stored energy estimated from TEM measurements, a good linear fit over wide strain range (shown in red in Fig. 4), though with some scatter, is found for the plot of $\sigma_{0.2 \%}$ versus $E_{\mathrm{S}}^{0.5}$. In contrast, the DSC-measured stored energy does not show a consistent linear relationship between $\sigma_{0.2 \%}$ and $E_{\mathrm{S}}^{0.5}$ over any extended range of strain.

To understand this difference, considering the comment above that the DSC measurements of stored energy should be more reliable, it should be noted that in Eq. (10) the flow stress is assumed to arise from dislocations that are only weakly stress-screened. The stored energy as estimated from TEM measurements can be assumed therefore to represent well a dislocation density that contributes directly to the flow stress (hence the linear relationship between $E_{\mathrm{S}}^{0.5}$ and $\sigma_{0.2 \%}$ ). The different relationship between $E_{\mathrm{S}}^{0.5}$ and $\sigma_{0.2 \%}$ found for the DSC data may therefore be 


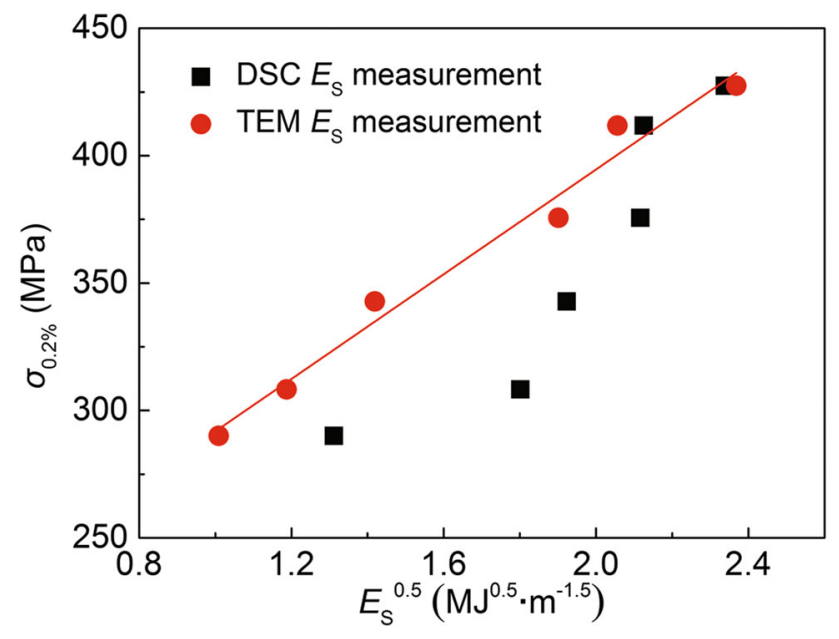

Fig. 4 Graph showing the relationship between $E_{\mathrm{S}}^{0.5}$ and the flow stress using both the DSC (black squares) and TEM (red circles) stored energy measurements. A linear fit to the TEM data is also given

attributed to the effect of balanced internal stresses within the microstructure. These will not affect the flow stress, but will contribute directly to the stored energy of deformation. As stress screening of dislocation boundaries increases with increasing strain, a smaller range of balanced internal stresses is expected with increasing strain, accounting for the observed decrease in the difference in stored energy estimated from the TEM measurements and determined from the DSC data.

\section{Conclusions}

The stored energy of cold-rolled copper has been investigated over a wide range of strain from $\varepsilon_{\mathrm{vM}}=0.42$ to $\varepsilon_{\mathrm{vM}}=5.21$ using DSC measurements and estimates based on local measurements of the microstructure made in the TEM. For both methods, a linear increase in stored energy with strain is found. The maximum difference in stored energy is factor of nearly 2.4 , and at the highest strains, the values based on TEM microstructural measurements approach the DSC-determined values, giving confidence to the absolute values of stored energy obtained. The ratio between the DSC and TEM-estimated values varies significantly however, which may lead to complications in estimation of local variations in stored energy within a sample deformed to a given nominal strain (i.e., for characterization of stored energy variations associated with deformation microstructure heterogeneities). Comparison of the stored energy values with the flow stress suggests that the TEM microstructure measurements capture well the dislocation density content responsible for the flow stress, but that the DSC technique additionally measures other contributions to the stored energy, such as the presence of balanced internal stresses.

Acknowledgments The authors gratefully acknowledge the support from the Danish National Research Foundation (No. DNRF86-5) and the National Natural Science Foundation of China (Nos. 51261130091 and 51171085) to the Danish-Chinese Center for Nanometals.

\section{References}

[1] M.B. Bever, D.L. Holt, A.L. Titchener, Prog. Mater. Sci. 17, 5 (1973)

[2] J.W. Martin, R. Doherty, B. Cantor, Stability of Microstructure in Metallic Systems, 2nd edn. (Cambridge University Press, Cambridge, 1997), pp. 147-166

[3] N. Hansen, Metall. Mater. Trans. A 32, 2917 (2001)

[4] N. Hansen, D.J. Jensen, Mater. Sci. Technol. 27, 1229 (2011)

[5] X. Huang, N. Hansen, Scr. Mater. 37, 1 (1997)

[6] X. Huang, G. Winther, Philos. Mag. 83, 5189 (2007)

[7] A. Godfrey, W.Q. Cao, N. Hansen, Q. Liu, Metall. Mater. Trans. A 36, 2371 (2005)

[8] O.V. Mishin, A. Godfrey, Metall. Mater. Trans. A 39, 2923 (2008)

[9] J. Hjelen, R. Orsund, E. Nes, Acta Metall. Mater. 39, 1377 (1991)

[10] H. Paul, J.H. Driver, Z. Jasienski, Acta Mater. 50, 815 (2002)

[11] E. Woldt, D. Juul Jensen, Metall. Mater. Trans. A 26, 1717 (1995)

[12] T. Ungar, E. Schafler, P. Hanak, S. Bernstorff, M. Zehetbauer, Mater. Sci. Eng. A 462, 398 (2007)

[13] A. Borbely, J.H. Driver, T. Ungar, Acta Mater. 48, 2005 (2000)

[14] A. Godfrey, N. Hansen, D. Juul Jensen, Metall. Mater. Trans. A 38, 2329 (2007)

[15] S. Goel, R. Jayaganthan, I.V. Singh, D. Srivastava, G.K. Dey, N. Saibaba, Acta Metall. Sin. (Engl. Lett.) 28, 837 (2015)

[16] M.C. Demirel, B.S. El-Dasher, B.L. Adams, A.D. Rollett, Electron backscatter diffraction, in Materials Science, ed. by A.J. Schwartz, M. Kumar, B.L. Adams, D.P. Field (Plenum, New York, 2000), pp. 65-74

[17] A.A. Benzerga, Y. Brechet, A. Needleman, E. Van der Giessen, Acta Mater. 53, 4765 (2005)

[18] T. Knudsen, W.Q. Cao, A. Godfrey, Q. Liu, N. Hansen, Metall. Mater. Trans. A 39, 430 (2008)

[19] A. Godfrey, Q. Liu, Scr. Mater. 60, 1050 (2009)

[20] D.A. Hughes, N. Hansen, Acta Mater. 48, 2985 (2000)

[21] Q. Liu, X. Huang, D.J. Lloyd, N. Hansen, Acta Mater. 50, 3789 (2002)

[22] Q. Liu, Ultramicroscopy 60, 81 (1995)

[23] W.T. Read, W. Shockley, Phys. Rev. 78, 275 (1950)

[24] A. Godfrey, D.A. Hughes, Mater. Charact. 48, 89 (2002)

[25] Z. Zhang, H. Xu, S. Wu, Y. Liu, Acta Metall. Sin. (Engl. Lett.) 26, 340 (2013) 